\title{
Comparison of three cell cycle associated antigens as markers of proliferative activity and prognosis in breast carcinoma
}

\author{
C E Gillett, D M Barnes, R S Camplejohn
}

\begin{abstract}
The staining patterns obtained with two antibodies against proliferating cell nuclear antigen (PC10 and 19A2) and another cell cycle associated antibody (KiS1) were compared with each other and with a number of established prognostic markers of breast carcinoma.

Although PC10 and 19A2 staining patterns were similar, only the latter was significantly associated with KiS1 antibody staining. These findings suggest that the two PCNA antibodies detect different epitopes.

KiS1 was the only antibody to show an association with $S$ phase fraction measured by flow cytometry $(p<0.001)$. It was also associated with histological grade $(p=0.003)$, oestrogen receptors $(p$ $=0.045)$, and DNA index $(p=0.007)$. PC10 showed no association with any of the markers of prognosis, while 19A2 was associated with histological grade $(p=$ $0 \cdot 017)$ and oestrogen receptors (p $=$ 0.043).

The two PCNA antibodies do not seem to be of value in measuring proliferative activity nor do they seem to be associated with established markers of prognosis in breast cancer.
\end{abstract}

(F Clin Pathol 1993;46:1126-1128)

Antibodies PC10 and 19A2 are both directed against proliferating cell nuclear antigen (PCNA), a 36 kilodalton protein essential for DNA synthesis. PCNA is synthesised in cells as they pass through $G_{1}$ and $S$ phases of the cell cycle. The protein has a long half life $(20$ hours), however, and is therefore detectable throughout the cell cycle. PCNA exists in at least two forms, one of which is tightly bound to replisomes or sites of DNA repair, and a second which is diffusely distributed throughout the nucleoplasm. This second form is easily extracted-for example, by treatment with detergent. ${ }^{1}$ This means that staining for the antigen is never entirely absent but shows an increase above the basal level during late $G_{1}$ and $S$ phases.

Antibody KiS1 is directed against a 160 kilodalton cell cycle associated nuclear protein. The antigen is also present throughout all phases of the cell cycle, although its precise role has yet to be identified. Concentrations are high immediately before mitosis. $^{2}$

\section{Methods}

Fifty patients with primary breast cancer diagnosed in 1990 at the ICRF Clinical Oncology Unit, Guy's Hospital, were included in the study. All tumour blocks were fixed in cold formol-saline, which optimally preserves the PCNA protein (unpublished observation).

Sections, $3 \mu \mathrm{m}$, were cut from each block and the peroxidase conjugated, streptavidin biotin complex method used to demonstrate antigen-antibody binding.

Antibody PC10 was donated by Professor $D$ Lane (University of Dundee) and used at a dilution of one in 200 overnight at $4^{\circ} \mathrm{C}$. Antibody 19A2 was a gift from Dr W Bolton (Coulter Immunology) and applied neat for one hour at room temperature, in accordance with the instructions. Antibody KiS1 was donated by $\mathrm{Dr} \mathrm{H}$ Kreipe (Kiel University, Germany) and used at a dilution of one in 100000 for one hour at room temperature.

A semiquantitative method of assessment was used to evaluate the staining. The proportion of cells staining was estimated as under $25 \%, 25-50 \%, 51-75 \%$ and more than $75 \%$ and scored as one to four, respectively. The intensity of staining was estimated as weak, moderate, or strong and scored as one to three, respectively. The cumulative proportion and intensity values provided the overall staining score. This method of assessment was undertaken independently by two of the authors to establish the interobserver reproducibility.

Staining scores for each antibody were compared with histological grade, lymph node status, oestrogen and progesterone receptor status (ER and PR), DNA index, and $S$ phase fraction (SPF) by flow cytometry, using a rank correlation test.

\section{Results}

PC10 staining was predominantly restricted to the nuclei of the epithelial cells. Mitotic figures showed no staining, although there was some weak staining of the cytoplasm. There was minimal staining of the stromal matrix (fig 1A).

Antibody 19A2 had a more intense nuclear staining pattern with a higher proportion of stained cells than PC10, but mitotic figures and the cytoplasm of interphase cells also stained. Furthermore, both the nuclei and cytoplasm of fibroblasts in the stroma were intensely stained (fig 1B).

KiS1 staining was predominantly restricted 

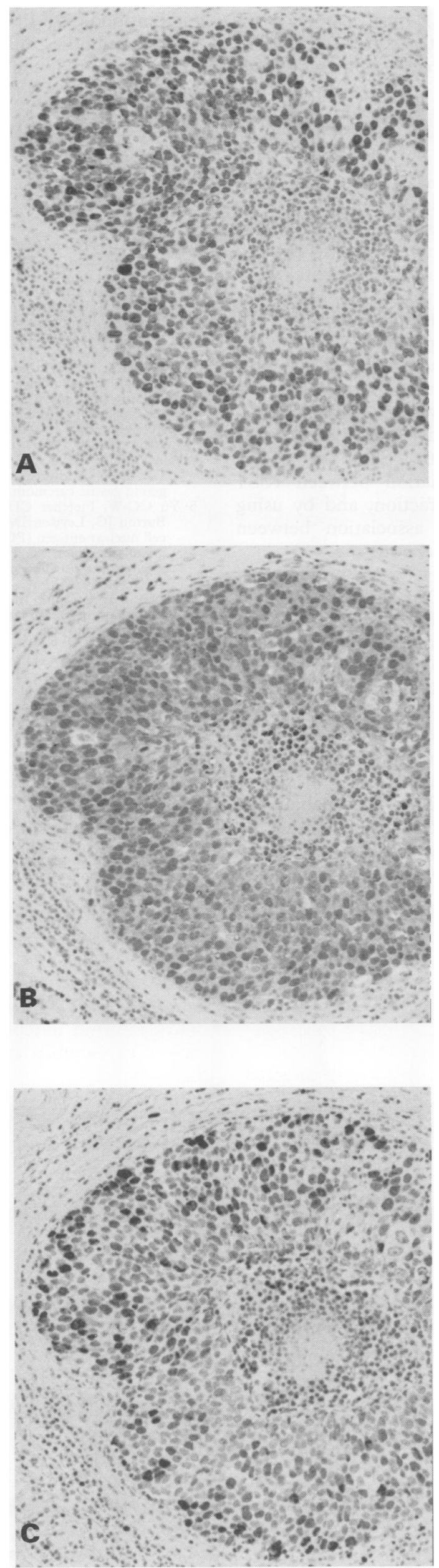

Figure 1 In situ ductal carcinoma of the breast showing (A) PC10, (B) 19A2, and (C) KiS1 immunostaining.

\begin{tabular}{|lll|}
\hline PC10 & 19A2 & KiS1 \\
None & Grade & Grade \\
& $p=0.017$ & $p=0.003$ \\
& Oestrogen & Oestrogen \\
& receptor & receptor \\
& $p=0.043$ & $p=0.045$ \\
& & DNA index \\
& & $p=0.007$ \\
& & S phase fraction \\
& & $p<0.001$
\end{tabular}

Prognostic features studied: histological grade; nodal status; estrogen receptor; progesterone receptor; DNA index and S phase fraction.

Figure 2 Significant associations between antibody staining score and prognostic factors.

to the nuclei of epithelial cells, with only a small amount of cytoplasmic staining. Mitotic figures often showed intense staining. There was minimal staining of the stromal matrix (fig 1C).

The semiquantitative method of assessment showed very good reproducibility between the two observers (Pearson's correlation coefficient; $r=0.707 ; p<0.001$ ).

The comparison of staining scores for the different antibodies produced conflicting data. Although PC10 scores were lower than those for 19A2, the two were highly significantly correlated $(p<0.001)$. KiS1 scores were lower than both the above, but there was a strong association between $\mathrm{KiS1}$ and 19A2 scores and no significant association between KiS1 and PC10 scores.

The correlations between antibody staining score to $\mathrm{S}$ phase fraction, DNA ploidy, and the prognostic markers assessed are shown in fig 2 .

\section{Discussion}

The three antibodies assessed in this study were all originally considered to detect proliferating cells. When their staining scores were compared with each other, however, there was no universal association. These results suggest that the two PCNA antibodies are directed against different epitopes, which are not necessarily expressed at the same time, and that KiS1 is detecting something different again.

PC10 staining has been shown to be of prognostic value in a variety of tumour types. ${ }^{3-5}$ In this study of breast carcinoma, however, in agreement with several other studies, PC10 staining showed no significant association with any of the markers of prognosis or with proliferative activity, as measured by flow cytometry. ${ }^{67}$

Antibody 19A2 has been reported by Battersby $e t a l^{8}$ to be strongly associated with proliferative activity when measured by thymidine labelling. In our study 19A2 staining score was significantly associated with histological grade and oestrogen receptor states, both considered to be markers of differentiation rather than markers of proliferative activity (even though one of the components of 
grade is a mitotic count). Furthermore, staining was not significantly correlated with $S$ phase fraction, a well accepted marker of proliferation.

In several cases both PC10 and 19A2 had a high staining score (5-7) when only a small percentage of cells were in S-phase. Our findings in breast carcinoma support data from other groups which have shown that PCNA can be expressed by cells which are not proliferating. This may be in response to the cell undergoing DNA repair. Experimental work by Hall et $a l^{9}$ has shown that PCNA expression can be induced by growth factors without proliferation taking place.

The antigen recognised by the KiS1 antibody has yet to be characterised, but in this study its expression showed a significant correlation with proliferative activity, as measured by flow cytometry. Sampson et al ${ }^{10}$ noted a similar association between $\mathrm{KiS} 1$ staining and $S$ phase fraction, and by using log rank analysis, an association between KiS1 staining and clinical outcome.

We have shown that the "proliferation associated markers" PC10 and 19A2 do not reliably demonstrate proliferative activity in breast carcinomas, compared with flow cytometry. This is probably the result of the complex nature of PCNA and its long half life. Hence the initial enthusiasm for these antibodies as prognostic indicators seems to have been misplaced as far as the study of breast disease is concerned.

KiS1 is one of several newly available antibodies which is also thought to show the presence of proliferating cells. To avoid any recurrence of the disappointment associated with antibodies to PCNA, however, KiS1 and similar antibodies must be carefully evaluated before they are launched as useful markers of breast cancer prognosis.

We thank Dr Rosemary Millis for the histological typing and grading of the tumours.

1 Wilson GD, Camplejohn RS, Martindale CA, Brock A, Lane DP, Barnes DM. Flow cytometric characterisation of proliferating cell nuclear antigen using the monoclonal antibody PC10. Eur 7 Cancer 1992;28A:2010-7.

2 Camplejohn RS, Brock A, Barnes DM, et al. Ki-S1, a novel proliferative marker: flow cytometric assessment of staining in human breast carcinoma cells. $\mathrm{Br} f$ Cancer of staining in human

3 Woods AL, Hall PA, Shepherd NA, et al. The assessment of proliferating nuclear cell antigen (PCNA) immunostaining in primary gastrointestinal lymphomas and its relationship to histological grade, $S+G_{2}+M$ phase fraction (flow cytometric analysis) and prognosis. Histopathology 1991;19:21-7.

4 Jain S, Filipe MI, Hall PA, Waseem N, Lane DP, Levison DA. Prognostic value of proliferating cell nuclear antigen in gastric carcinoma. $\Im$ Clin Pathol 1991;44:655-9.

5 Yu CC-W, Fletcher CDM, Newman PL, Goodlad JR, Burton JC, Levison DA. A comparison of proliferating cell nuclear antigen (PCNA) immunostaining, nucleolar cell nuclear antigen (PCNA) immunostaining, nucleolar
organiser region (AgNOR) staining and histological organiser region (AgNOR) staining and histological grading in gastroint

6 Leonardi E, Girlando S, Serio G, et al. PCNA and Ki67 expression in breast carcinoma: Correlations with clinical and biological variables. F Clin Pathol 1992;45. 416-9.

7 Gasparini G, Meli S, Pozza F, Cazzavillan S, Bevilacqua P. PC-10 antibody to Proliferating Cell Nuclear Antigen (PCNA) is not related to prognosis in human breast carcinoma. Growth Regulation 1992;2:145-50.

8 Battersby A, Anderson TJ. Correlation of proliferative activity in breast tissue using PCNA/cyclin. Hum Pathol activity in breast

9 Hall PA, Coates PJ, Goodlad RA, Hart IR, Lane DP. Proliferating cell nuclear antigen expression in noncycling cells may be induced by growth factors in vivo. $\operatorname{Br} \mathcal{F}$ Cancer (in press)

10 Sampson SA, Kreipe H, Gillett CE, et al. KiS1-A novel monoclonal antibody which recognises proliferating cells: Evaluation of its relationship to prognosis in mammary carcinoma. $\mathcal{f}$ Pathol 1992;168:179-85. 